\title{
NON-LINE ANALYSIS OF STIFFNESS IN COMPRESSION CONDITIONS
}

\author{
Maciej Kahsin* \\ Gdańsk University of Technology, Poland \\ Dawid Stecki \\ Gdańsk Shiprepair Yard „Remontowa” S.A., Poland
}

\begin{abstract}
The analyzes were aimed at demonstrating the influence of parameters describing the deformation of the structure on the uncertainty of critical force, and the impact of technological imperfections on stress uncertainty in compression conditions. In a linear buckling analysis, the problem is considered only for the initial, permanent state of the stiffness matrix. In the case of demonstrating the influence of initial deformations on the behavior of the structure under load, it is necessary to visualize changes in stiffness over time. To this end, a non-linear MES analysis was carried out, which will take into account local changes in the stiffness of the model through a gradual increase in the load. Thus, the difference in stiffness is taken into account, which in the linear problem is infinite. The analysis was used to examine the local and global sensitivity of the parameters describing: plating thickness as well as deformation caused by the technological process on the stress value reduced by Huber hypothesis, and the value of normal stress. To take into account the influence of non-specified values of the magnitude of geometric deviations, and their simultaneous influence on the range of obtained results, the Experimental Planning Method and the Surface Method of Answers were used.
\end{abstract}

Keywords: Numerical simulations, Uncertainty of model parameters, Reliability of results, Statistical approach, Limit load

\section{INTRODUCTION}

Each computer simulation that uses the Finite Element Method requires an unambiguous description of the calculated system of equations (i.e. a monovalent quantity for a given parameter in each potential computational iteration). Such a state causes that each time a strictly determined result assigned to a specific set of strictly defined values of the model parameters is obtained. A question arises as to how much such a result is reliable, and hence useful in assessing the actual state of the structure being built. This issue is particularly important in the case of marine or coastal constructions, because usually: enormous size, complexity of the structure, methods of combining components, etc., make it impossible to create a fully virtual reality virtual model. One of the possible solutions for assessing the quality of the virtual model is its comparison with the results of measurements performed on the real object [7]. If such data is lacking, one can use the results of statistical analysis carried out on a series of numerical simulation results performed for a given, but variable range of input parameter values. In order to estimate the impact of changes in the value of the input parameter on the quantitative change in the result value, the Experimental Planning Method and the Surface Response Method were used in the work. In order to investigate whether the impact of the variability of the numerical model parameters on the changes in the values of the results obtained can be assessed qualitatively, the local and global sensitivity of the model was determined. 


\section{DESCRIPTION OF THE EXAMINED STRUCTURE}

The analyzed structure consists of a section of the bottom structure of the ship, i.e. one stiffening and plating. The model span is two frame spacings $(1 / 2+1+1 / 2)$ in the longitudinal direction, while in the transverse direction there is one stiffener spacing. The same dimensions of the structural elements were used as the base, i.e. the plating thickness $\mathrm{t}=12 \mathrm{~mm}$, and the stiffening is an angle $\mathrm{L} 230 \times 11 \times 110 \times 13$. The material used is category A normal strength steel with a yield strength $\mathrm{Re}=235 \mathrm{MPa}$. Young's module is $\mathrm{E}=206 \mathrm{GPa}$, Poisson's number is $\mathrm{v}=0,3$.

The analysis focuses on one stiffening, because it will allow to more reliably measure the impact of input parameters on the output size. In addition, it has simplified parameterization of variables describing the uncertainties associated with the production process.

The calculation model takes into account the parameters describing the uncertainties in the structure. They concern the geometrical dimensions of individual elements, possible deformations arising after welding of elements and material. In addition, assembly errors were also taken into account, which affect the spacing of stiffeners and their span. The occurrence of welding deformations is closely related to this technology of joining structural elements. The form of deformations largely depends on the method used and its parameters [2]. Individual defects occurring in the construction caused both by welding distortions and assembly errors were modeled on the basis of the available information publication of the Polish Register of Shipping - Shipbuilding and repair standards (Table 1) [13]. Limit values (maximum) have been adopted in the model. The deformation of the flange was modeled as the angle between the flange and the web of the stiffener, so that the extreme values correspond to the accepted range.

Tab. 1. Parameters describing technological deformations

\begin{tabular}{|l|c|c|c|}
\hline & Parameter & Range & Stat. values \\
\hline $\begin{array}{l}\text { Deformation } \\
\text { of the gunwales }\end{array}$ & Dmoc & $88,15 \div 91,85^{\circ}$ & $\begin{array}{c}\mu=90^{\circ} \\
\sigma=0,6^{\circ}\end{array}$ \\
\hline $\begin{array}{l}\text { Deformation } \\
\text { of the web }\end{array}$ & Dsrod & $0 \div 18 \mathrm{~mm}$ & $\begin{array}{c}\mu=9 \mathrm{~mm} \\
\sigma=2,7 \mathrm{~mm}\end{array}$ \\
\hline $\begin{array}{l}\text { Stiffening } \\
\text { deformation }\end{array}$ & Duszt & $0 \div 12 \mathrm{~mm}$ & $\begin{array}{c}\mu=6 \mathrm{~mm} \\
\sigma=1,8 \mathrm{~mm}\end{array}$ \\
\hline $\begin{array}{l}\text { Plating } \\
\text { deformation }\end{array}$ & Dp & $0 \div 16 \mathrm{~mm}$ & $\begin{array}{c}\mu=8 \mathrm{~mm} \\
\sigma=2,4 \mathrm{~mm}\end{array}$ \\
\hline
\end{tabular}

Deformations were modeled in a simplified manner so that the central portion of the stiffeners located between the frames of the framework were deformed. A parameterized, varying cross-section of stiffening and plating was used in the middle of the distance between the frames of the framework (Figure 1). Deformation in the intermediate area changes linearly.

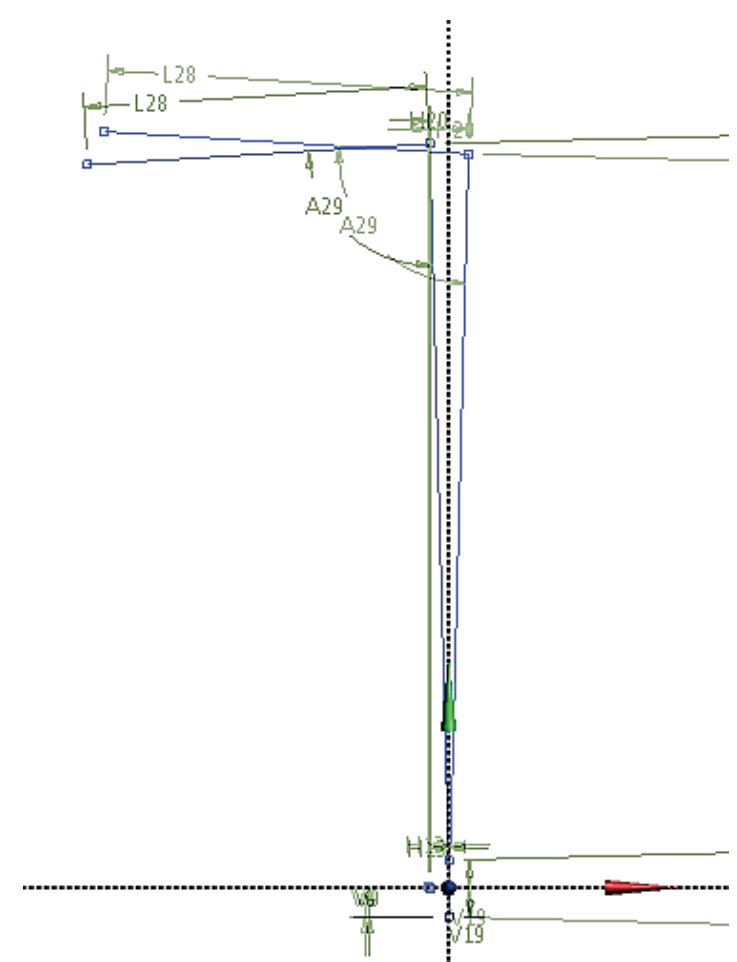

Fig. 1. Parameterized stiffening profile, extreme positions of the profile modelling deformations

\section{MES MODEL}

The analysis used a model from the previous study consisting of one longitudinal stiffening and plating (span $1 / 2+1+1 / 2$ ). When defining the MES model, the recommendations and methods given in the Technical Background Report for CSR [4] and the Recommended Practice Recommendations for the DNV classification society [4] were recommended. Relevant from the point of view of credibility, the results were to apply appropriate boundary conditions so that the stiffening and the plating belt retained the form of buckling consistent with the results of the linear buckling analysis. The conditions used were based on the recommendations given in the abovementioned sources (TBR for CSR and DNV). The boundary conditions of the MES model are shown in Figure 2.

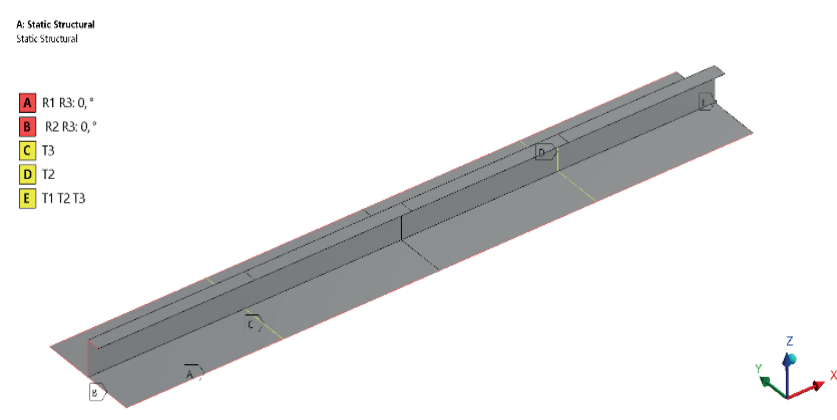

Fig. 2. Boundary conditions of the non-linear MES model 
The structure was loaded in a stepped way so as to be able to track changes in the stiffness of the model, and to realize nonlinearities, both material and geometric. The compressive load has been applied to the transverse edge of the model. A force equal to $\mathrm{F}=2250 \mathrm{kN}$ was applied.

A dense division into finite elements was applied, keeping the proportions of the sides as close as possible to the ratio of the edge length of $1: 1$. The analysis includes parameters regarding the thickness of the Tp plating and technological deformations: Dsrod, Duszt, Dmoc and Dp. Plating thickness was included to reference the sensitivity to the previous analysis. In order to illustrate the influence of input parameters on the uncertainty of the output quantity, the same distributions and statistical measures were used as in the previous analysis. As the initial values, the maximum stress was determined according to Huber hypothesis and maximum normal stress.

\section{RESULTS OF NUMERICAL ANALYSIS}

In Fig. 3 and Fig. 4, an exemplary result of the MES analysis was shown. As one can see the form of deformation of the structure is consistent with the form of loss of stability, which indicates the correctness of the adopted methodology.

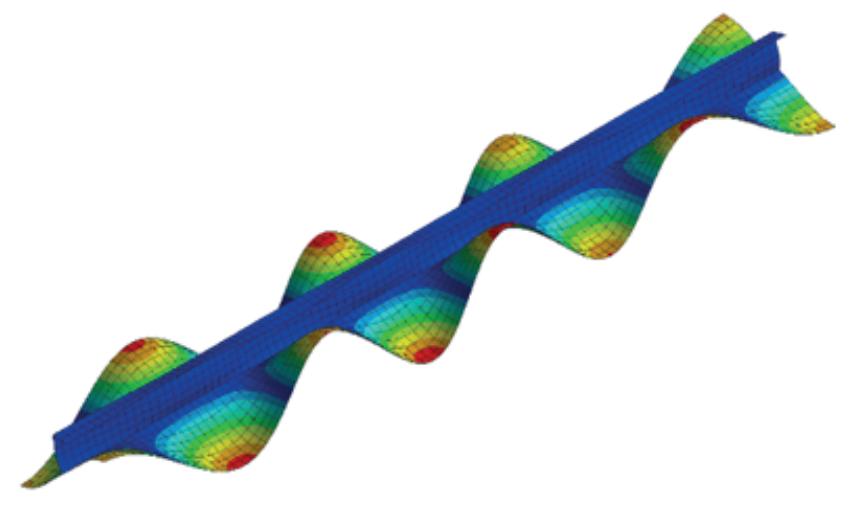

Fig. 3. Obtained buckling forms of the MES model-linear model

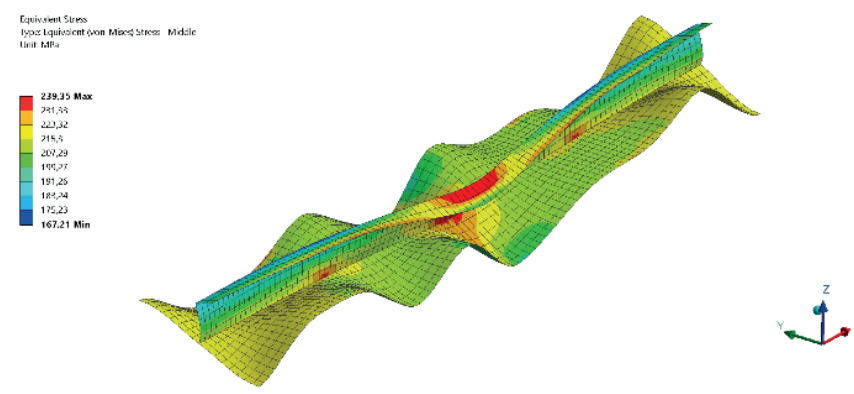

Fig. 4. Obtained buckling forms of the MES model - non-linear model

Due to the complexity of the problem under consideration and possible strong non-linearity between input parameters and output values, Optimal Space Filling was used as the method of experimental planning [12]. It optimally fills the tested space according to the set criterion. In essence, this method is based on the operation of the Latin hypercubes plan. First, points are generated in the same way as in the LHS method. Then the points are subjected to several optimizations so as to obtain their even distribution by maximizing the distance between them. The OSF algorithm thus enables to achieve maximum insight into the analyzed space while maintaining fewer experimental points. This method has similar properties to the LHS method. Extreme points are not always included, and too few experimental points result in poor quality results. With increasing the number of experiments, this method provides better quality results and provides a much better representation of the examined space. Fig. 5 shows an example of the levels of the values of the Tp and Dmoc parameters in the experiment plan.

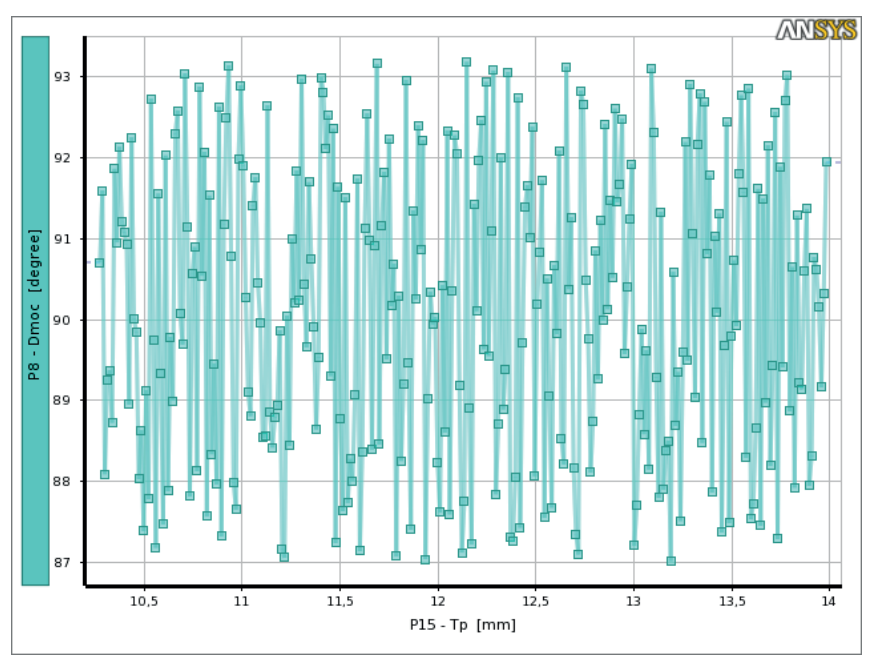

Fig. 5. Levels of Tp and Dmoc parameters values in the experiment plan

A genetic algorithm was used again to generate the answer surface [15]. The genetic algorithm is not in itself a surface response method, but can be used to automate the process of selecting, configuring and generating different types of hem. This algorithm aims to create a response surface that is best suited to your analysis.

First, a population of different response areas is created that are generated in parallel. For each of them, the matching functions determine which will be the most efficient. The accuracy of reproduction of experimental points and stability is taken into account. The genetic algorithm can create an answer surface using one of the known methods or generate a combination of several different surfaces obtained from the crossover operation.

The answer surface obtained using the genetic algorithm can be written as (1):

$$
\hat{y}_{c}(x)=\sum_{i=1}^{N} w_{i} \cdot \hat{y}_{i}(x)
$$

where: $\hat{y}_{c}$ is the whole forecast, $\hat{y}_{i}$ is the $i$-th forecast of the response, $N$ is the number of metamodels used, and $w_{i}$ is the weighting factor of the $i$-th area of the answer. 
In order to estimate the best values of the weighting factor at experimental points, it is possible to minimize the mean square error and perform cross-validation [16]. Crossvalidation methods include, among others Leave-One-Out and $\mathrm{K}$-fold validation.

The initially determined population of the response surface is subjected to operations typical of the genetic algorithm, such as crossbreeding and mutation. When crossing, there are two types of operations. The first involves crossing between surfaces of the same type (e.g. Kriging) by exchanging only part of the property from the first "parent" to the second, e.g., the type of nuclear functions (kernel) between two kriging surfaces is exchanged. The second crossed case creates a new response surface, which is a combination of two different "parents" surfaces, e.g. from a combination of kriging and linear regression surfaces. The mutation provides a variety of response surfaces. The algorithm in this case removes part of the surface of a given type, which occurs too much and retains other surfaces occurring in a smaller amount. Properties of the generated response areas for both output quantities are summarized in Table 2, while the goodness of the fit in relation to the experimental points and verification points is shown in Table 3.

Tab. 2. Answer areas

\begin{tabular}{|c|c|c|}
\hline \multirow[t]{3}{*}{$\begin{array}{l}\text { Output } \\
\text { data }\end{array}$} & The type of answer surface & Properties \\
\hline & \multicolumn{2}{|c|}{ Combination of 5 members: } \\
\hline & 1. Support Vector Regression & Weight: 0,1961 \\
\hline \multirow{4}{*}{$\sigma_{\text {red }}$} & 2. Kriging & Weight: 0,36283 \\
\hline & 3. Support Vector Regression & Weight: 0,17253 \\
\hline & 4. Support Vector Regression & Weight: 0,17834 \\
\hline & 5. Polynomial regression & $\begin{array}{l}\text { Weight: 0,09021; Square } \\
\text { polynomial }\end{array}$ \\
\hline \multirow{3}{*}{$\sigma_{N}$} & \multicolumn{2}{|l|}{ Combination of 2 members: } \\
\hline & 1. Support Vector Regression & Weight: 0,3239 \\
\hline & 2. Kriging & Weight: 0,6761 \\
\hline
\end{tabular}

Tab. 3. Goodness of matching the answer surface

\begin{tabular}{|l|c|c|}
\hline Experimental points & $\sigma_{\text {red }}$ & $\sigma_{N}$ \\
\hline Coefficient of determination & 0,96 & 0,99 \\
\hline Root mean square error & 2,9 & 1,416 \\
\hline Maximum absolute error [\%] & 217,29 & 89,06 \\
\hline Average absolute error [\%] & 6,07 & 3,03 \\
\hline Verification points & 4,49 & 5,25 \\
\hline Root mean square error & 34,3 & 51,4 \\
\hline Maximum absolute error [\%] & 29,9 & 29,47 \\
\hline Average absolute error [\%] & \multicolumn{2}{|l}{} \\
\hline
\end{tabular}

As a result of the conducted analysis, local sensitivity $[1,8]$ of the input parameters was obtained. The greatest influence on the uncertainty of the reduced stress (Fig. 6, Fig. 7.) has the thickness of the Tp plating and the plating deformation in the plane of the Dsrod web. The sensitivity of these parameters is $-36 \%$ and $-27 \%$, respectively. Negative values mean that when the given parameter increases, the output value decreases. The other parameters do not have such a significant impact on the stress uncertainty. Their sensitivity values are $-4,4 \%$ for Duszt, $-2,6 \%$ for Dmoc and 3,5\% for Dp.

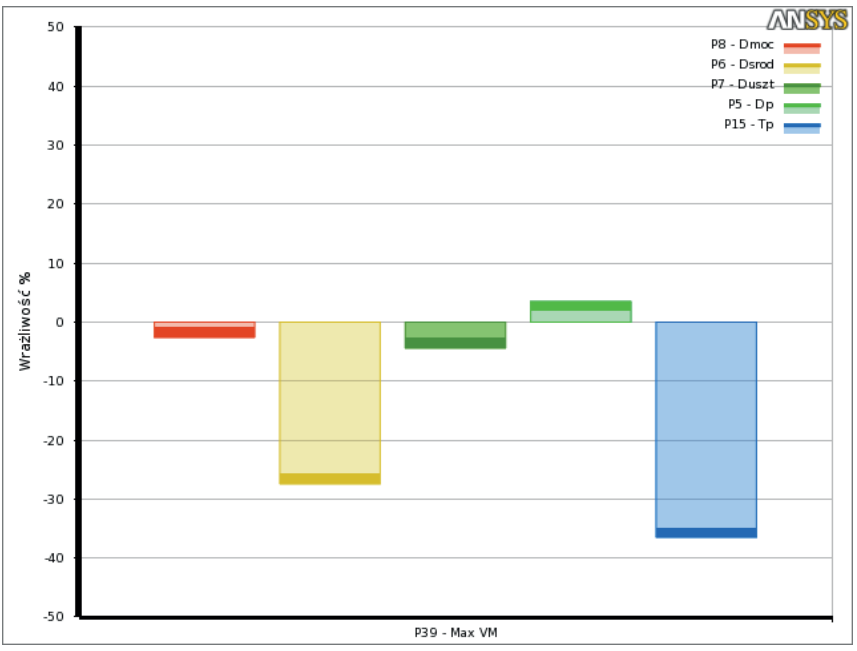

Fig. 6, Local sensitivity of input parameters relative to $\sigma H$

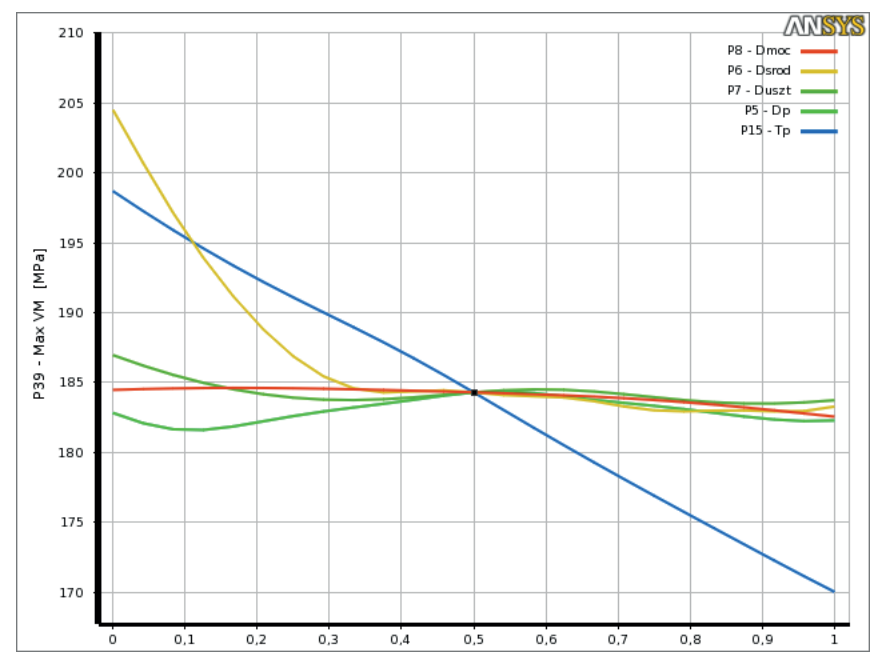

Fig. 7. The magnitude of influence of input parameters on $\sigma H$

The surface of the response $[5,9,11]$ showing the effect of the combination of Dsrod plating thickness and deformation parameters is shown in Fig. 8. The reduced stress is clearly increased when initially the plating in the plane of the stiffener web is deformed below the plane of the plating. A significant increase in stresses was also noted for the combination of parameters Dsrod and Dp (Fig. 9.) An interesting relationship is the effect of deformation of the plating halfway between stiffeners (Dp). For the Dsrod extreme value, the strain Dp can change the stress level reduced by $20 \mathrm{MPa}$. 


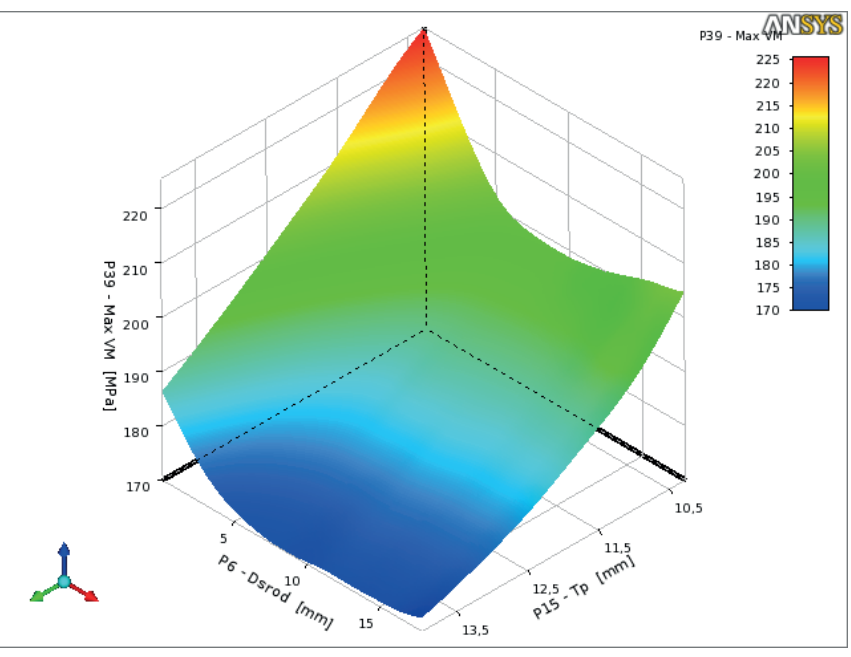

Fig. 8. Diagram of response surface - influence of Dsrod and Tp on $\sigma H$

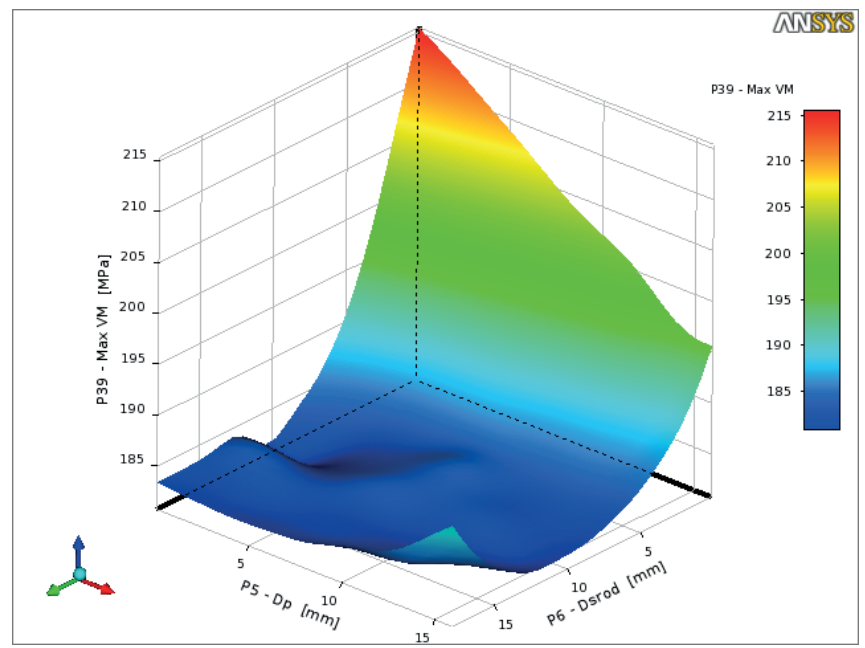

Fig. 9. Diagram of response surface - influence of Dsrod and Dp on $\sigma H$

The local sensitivity of the input parameters with reference to the uncertainty of normal stress is shown in Fig. 10. In this case, it can be seen that the greatest influence on the change in the stress value is the strain Dsrod, i.e. the deformation of the plating in the plane of the stiffener and plating thickness. Sensitivity values of these parameters are $37,5 \%$ and $35,7 \%$, respectively. There is also a greater than the reduced strain effect of strain $\mathrm{Dp}$, which is $6,5 \%$. The deformation of the Dmoc gunwale and the stiffness of the Duszt have a small effect on the initial size. The sensitivity is 3,2\% for Dmoc and $2,3 \%$ for Duszt. The impact of deformations is important only for the lower range of values, i.e. below the plane of the plating. This is illustrated in Fig. 11. The combinations of the parameters Tp and Dsrod as well as Dp and Dsrod presented in Fig. 12 and Fig. 13 show how the technological deformations have a significant influence on the uncertainty of the normal stress. Differences in stress levels reach 30-50 MPa.

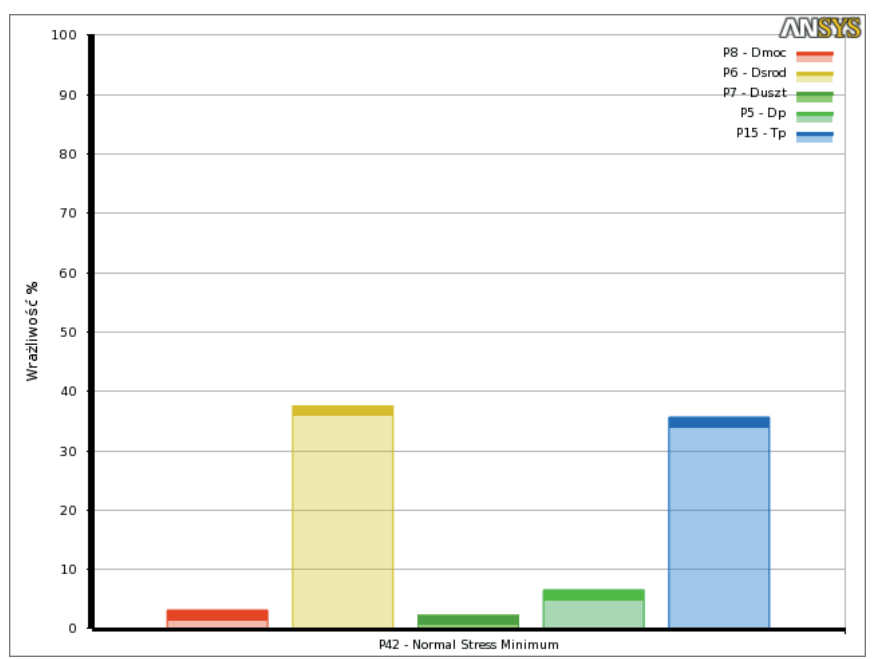

Fig. 10. Local sensitivity of input parameters to $\sigma N$

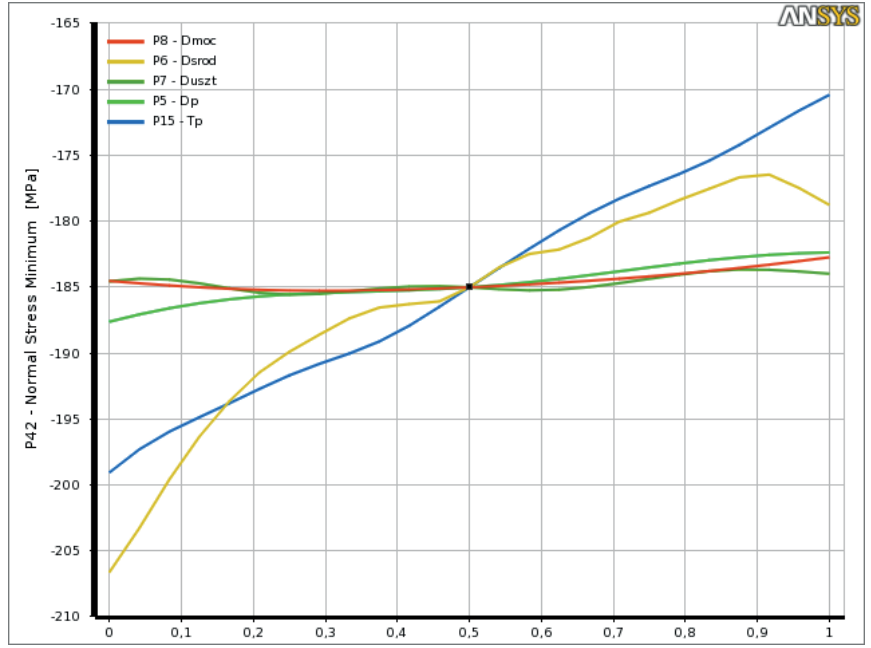

Fig. 11. The size of the impact of input parameters on $\sigma N$

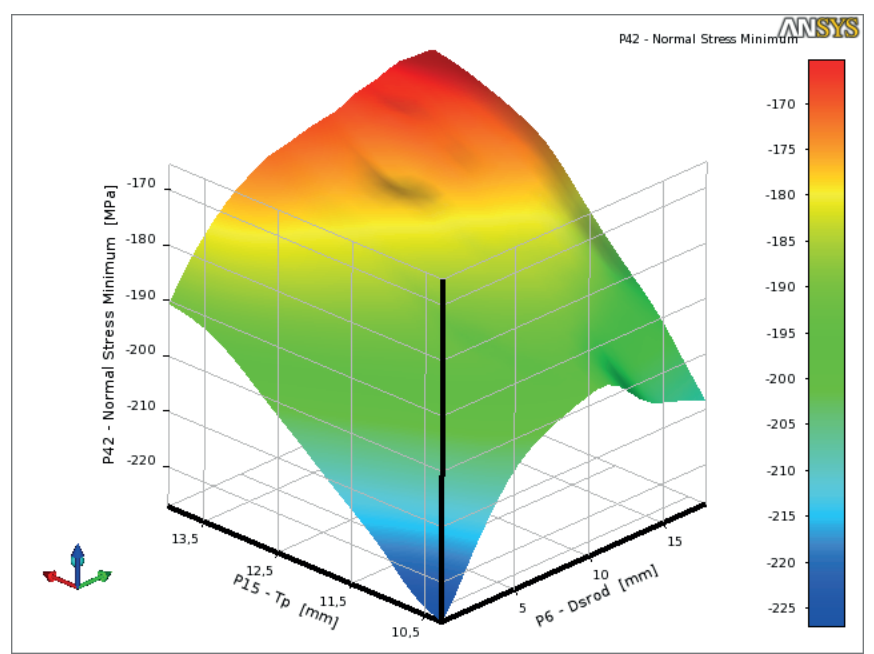

Fig. 12. Response area diagram - the influence of Tp and Dsrod on $\sigma N$ 


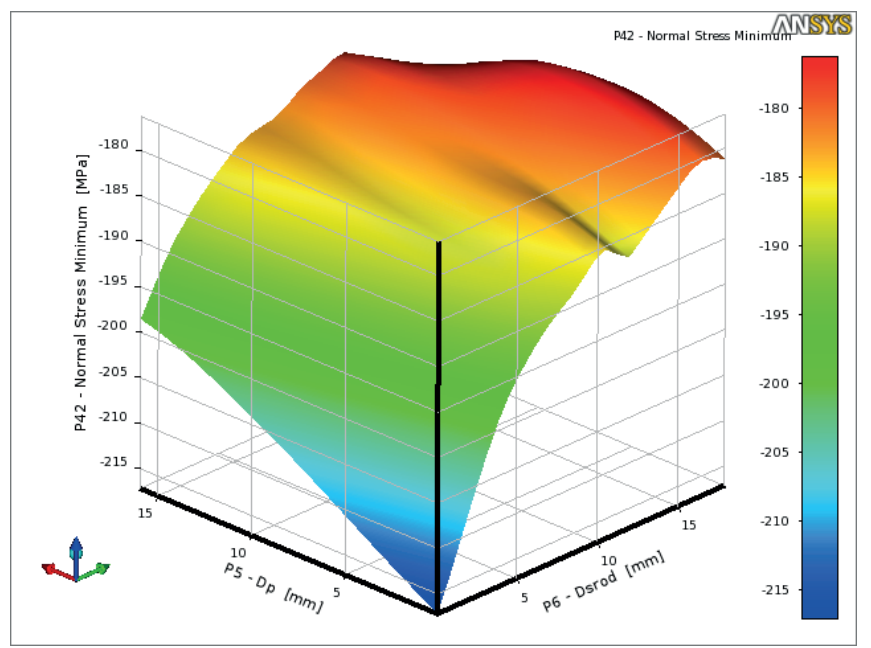

Fig. 13. Response surface diagram - the effect of Dsrod and Dp on $\sigma N$

The analysis also determined the global sensitivity [3, 9, 14] which is based on the probability of deviation of a given parameter. 100,000 samples were generated using the LHS method. The global sensitivity of input parameters to the uncertainty of reduced stress and normal stress are shown in Fig. 14. and Fig. 15. respectively. It can be noticed that the uncertainty of the thickness of the plating has the greatest influence on the uncertainty of stress both reduced and normal. In the case of normal stress, the strain Dsrod has similar sensitivity as the deviations of the plating thickness. In the case of uncertainty of the reduced stress, the Dsrod deformation constitutes about $1 / 3$ of the effect of the plating thickness. The global sensitivity analysis also showed a noticeable effect of Dp deformation on the uncertainty of the normal stress value.

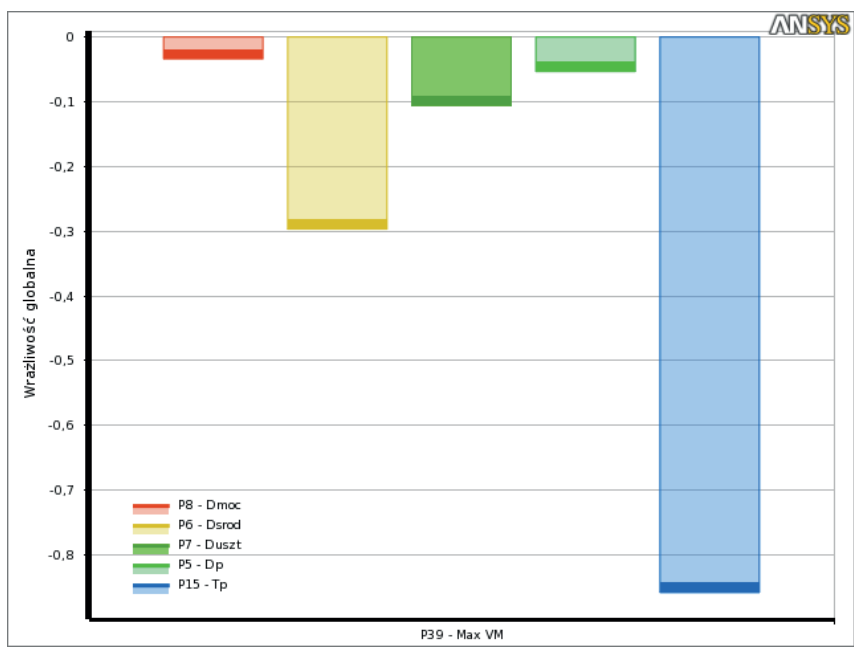

Fig. 14. Global sensitivity of parameters to $\sigma H$

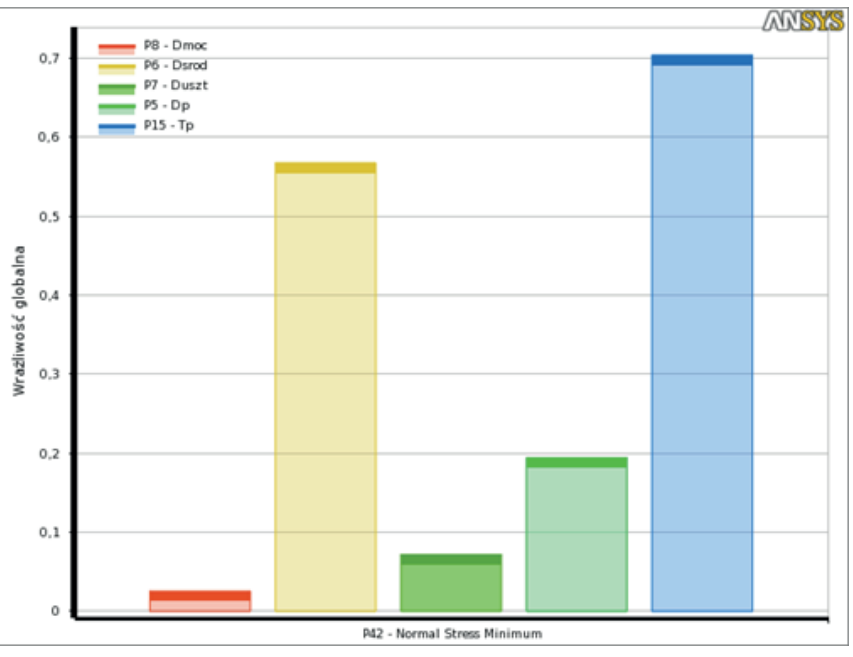

Fig. 15. Global sensitivity of parameters to $\sigma N$

In summary, the study examined the effect of technological deformations on reduced stresses and normal stresses in compression conditions. The analysis showed that deformations in the plane of the stiffener web (Dsrod) are significant from the point of view of the reduced and normal stress. In the case of normal stress, the plating deformation occurring in the middle of the distance between stiffeners is also significant. The dominant effect of plating thickness on the stress value in the structure demonstrated in previous analyzes was also confirmed.

\section{CONCLUSIONS}

The paper analyzes the impact of parameters of the finite element model on the structural response. It was used to examine the uncertainty in the model's response. Considered as uncertainty of input parameters, such as deviations in the dimensions of structural members, dimensional tolerances, and the technological process may affect the uncertainty of the output size of the model.

To determine local and global sensitivity, the analysis of MES and statistical methods were used. The research with the use of finite elements served as a research stand on a parameterized geometric model. Using the plan of experiments, an adequate number of static analyzes were carried out in order to obtain experimental points. Then, these points were approximated using the surface response method to find a functional relationship between the input and the model output. This correlation was next used to determine the sensitivity of the local and global model.

Sensitivity analysis was aimed at determining the impact of the uncertainty of input parameters on the uncertainty of the output. It was examined how deviations related to the dimensions of structural elements, material and technological deformations affect the uncertainty of critical force. In this analysis, LHS sampling was used to determine global sensitivity. The analysis was supposed to provide information 
on which elements of the construction process of flat sections should be paid attention to, in order to exclude their negative impact on the strength of the structure. To be able to model technological deformations, the tested model was limited to one stiffening and a plating belt with a span of 2 frame spacings.

In order to determine the effect of technological deformations on the values of reduced and normal stress under axial compression conditions, the types and boundary strain ranges were examined, which were considered acceptable in the shipbuilding standards. To determine these relationships, a non-linear MES analysis was used, taking into account large displacements and material non-linearity. The sensitivity test showed that the only deformation causing large deviations of the stress value is the deformation of the plating in the plane of the web of the element. Noticeable effect on normal stresses also has the deformation of the plating that occurs halfway between the stiffeners. It should be added that this impact is only relevant for negative deformations in the $\mathrm{z}$ direction.

The methods used can be a good tool for testing the sensitivity of the results of the numerical analysis of the virtual model, and can also be used to assess the impact of uncertainty on the quality of the obtained results. Combining techniques based on engineering calculations and statistical data provides many advantages. By performing the sensitivity analysis, the designer is able to examine a given project in many aspects, thus minimizing the need for prototyping. Therefore, he can more consciously make decisions related to the project. Sensitivity analysis is also used to test the uncertainty of the model's output data. It is a good tool when analyzing production processes and finding faults in them. Such an analysis may reveal often surprising connections and couplings between the uncertainties of the input parameters of the model.

\section{LITERATURE}

1. Broekea G. Voorna G., Ligtenbergb A.: Which Sensitivity Analysis Method Should I Use for My Agent-Based Model?. Journal of Artificial Societies and Social Simulation 19, 2016.

2. Chen, Z., Ajit Shenoi, R.: Influence of welding sequence on welding deformation and residual stress of a stiffened plate structure. Ocean Engineering, Volume 106, 15 September 2015, Pages 271-280.

3. Czitrom V.: One-Factor-at-a-Time Versus Designed Experiments, American Statistician. Vol. 53, 1999.

4. Det Norske Veritas, Recomended Practice: Determination of Structural Capacity by Non-linear FE analysis Methods, June 2013
5. Fisher R.A.: The Design of Experiments. Eighth Edition. Oliver and Boyd, Edinburgh 1966.

6. International Association of Classification Societies: Technical Background Report for CSR, 2016.

7. Kahsin M., Luczak M.: Numerical Model Quality Assessment of Offshore Wind Turbine Supporting Structure Based on Experimental Data. Structural health monitoring 2015: system reliability for verification and implementation, vols. 1 and 2, pp. 2817-2824 Published: 2015.

8. Kyung K. Choi, Nam H. Kim: Structural Sensitivity Analysis and Optimization 1. Mechanical engineering series, Berlin, Germany, 2013

9. Marino S., Hogue I.B., et al.: A Methodology For Performing Global Uncertainty And Sensitivity Analysis In Systems Biology, 2008

10. Mathews D.G.: Design and Analysis of Experiments, Eighth edition. Arizona State University, 2013.

11. Mathews D.G.: Design of Experiments with MINITAB. American Society for Quality, 2005.

12. Montgomery, D.C.: Design and Analysis of Experiments, Eighth edition.Arizona State University, 2013.

13. Polski Rejestr Statków, Standardy budowy i napraw statków. Publikacja informacyjna nr 16/I, Gdańsk 2011.

14. Saltelli A., Ratto M., Andres, et al.: Global Sensitivity Analysis: The Primer. John Wiley \& Sons. (2008).

15. Stocki R.: Analiza niezawodności $i$ optymalizacja odpornościowa. Instytut Podstawowych Problemów Techniki Polskiej Akademii Nauk, Warszawa 2010

16. Viana, F.A.C., Haftka R.T., Steffen V.: Multiple Surrogates: How Cross-Validation Errors Can Help Us to Obtain the Best Predictor. Structural and Multidisciplinary Optimization, Vol. 39, No. 4, 2009, pp. 439-457. 


\section{CONTACT WITH THE AUTHORS}

Maciej Kahsin

e-mail:mkahsin@pg.edu.pl

Gdańsk University of Technology

Faculty of Ocean Engineering and Ship Technology

11/12 Narutowicza St.

80-233 Gdańsk

Poland

Dawid Stecki

Gdańsk Shiprepair Yard „Remontowa” S.A.

Na Ostrowiu 1

80-958 Gdańsk

Poland 